\title{
Estudo comparativo: método empírico do DNIT e mecanístico- empírico (Medina) no dimensionamento de pavimentos flexíveis
}

O presente estudo de pesquisa fez uso do novo programa de dimensionamento de pavimentos flexíveis o Método de Dimensionamento Nacional - Medina programa totalmente brasileiro, desenvolvido por meio de parcerias entre entidades brasileiras. Como objetivo realizou-se uma análise comparativa entre os métodos de dimensionamento de pavimentos, o empírico atualmente empregado pelo Departamento Nacional de Infraestrutura e Transportes (DNIT) e o método mecanístico-empírico de Dimensionamento Nacional de Pavimentos (MEDINA), a fim de elucidar o uso do método mecanístico-empírico. Com a finalidade de comparar o dimensionamento das camadas de base e sub-base de pavimentos flexíveis, por meio dos diferentes métodos, sendo adotado o método empírico que tem como fundamento os dados de CBR, empregado pelo DNIT e o mecanístico-emprírico (Medina) com base em conceitos da teoria da elasticidade. Para cálculo das espessuras das camadas do pavimento flexível, foram propostos dois volumes de tráfego diferentes. Os dados dos materiais que foram utilizados nas camadas de base e sub-base foram obtidos a partir de estudos bibliográficos prévios. Na sequência foi realizado o dimensionamento do pavimento com os dois métodos propostos. Dentre os resultados obtidos, observa-se que Medina permite maiores análises e variações de materiais de revestimento, com base nas propriedades dos materiais. Para as possibilidades analisadas, destaca-se o tipo de material utilizado na camada do revestimento influenciando consideravelmente em sua espessura, fator relacionado ao módulo resiliente desses materiais, ou seja, para os casos propostos a espessura da camada de revestimento foi menor quanto maior foi o MR do revestimento adotado. O resultado que mais se destacou foi no caso $2.2 \mathrm{em}$ que o revestimento foi de concretos betuminoso com $7,5 \mathrm{~cm}$ de espessura no método empírico e pelo método mecanístico-empírico concreto asfáltico de classe 4 com 9,4cm manifestou melhores resultados para as camadas de revestimento, atendendo aos padrões de dimensionamento do programa. Ressalta-se que o dimensionamento efetuado pelo novo método colabora com a viabilidade a longo prazo e o pavimento tende a alcançar seu tempo de vida útil com maior segurança e economicidade, comparado ao método empírico.

Palavras-chave: Tráfego; Simulação de pavimentos; Espessuras; CBR; Módulo de Resiliência.

\section{Comparative study: empirical method of DNIT and mechanistic- empirical (Medina) in the design of flexible pavements}

The present research study made use of the new flexible sidewalk design program, the National Design Method - Medina, a totally Brazilian program developed through a partnership among Brazilian entities. A comparative analysis between the empirical sidewalk design methods currently used by the National Department of Infrastructure and Transportation (DNIT) and the mechanistic-empirical method of National Pavement Design (MEDINA) was performed in order to elucidate the use of the mechanistic-empirical method. In order to compare the design of the base and subgrade layers of flexible sidewalks, by means of the different methods, the empirical method was adopted, which is based on the CBR data used by DNIT, and the mechanistic-empirical method (Medina) based on concepts of the elasticity theory. To calculate the thicknesses of the flexible sidewalk layers, two different traffic volumes were proposed. The data of the materials that were used in the base and sub-base layers were obtained from previous literature studies. Subsequently, the sidewalk was designed using the two proposed methods. Among the results obtained, it can be observed that Medina allows greater analysis and variations of the surfacing materials, based on the properties of the materials. For the analyzed possibilities, the type of material used in the coating layer influences considerably its thickness, a factor related to the resilient modulus of these materials, i.e, for the proposed cases, the thickness of the coating layer was lower the higher the MR of the adopted coating. The result that stood out the most was in case 2.2, in which the coating was made of bituminous concrete $7.5 \mathrm{~cm}$ thick in the empirical method, and the mechanistic-empirical method, class 4 asphalt concrete $9.4 \mathrm{~cm}$ thick, showed better results for the coating layers, meeting the design standards of the program. It is noteworthy that the design made by the new method collaborates with the long-term viability and the sidewalk tends to reach its lifetime with greater safety and economy, compared to the empirical method.

Keywords: Traffic; Simulation of sidewalks; Thicknesses; CBR; Modulus of Resilience.

Topic: Engenharia Civil

Reviewed anonymously in the process of blind peer.
Received: $17 / 12 / 2020$

Approved: 10/03/2021
Larissa Macário da Silva

Instituto Tocantinense Presidente Antônio Carlos, Brasil

http://lattes.cnpq.br/4053474280236075

larissamacario184@gmail.com

Rafaella Oliveira Guimarães Santos

Instituto Tocantinense Presidente Antônio Carlos, Brasil

http://lattes.cnpq.br/2006870316534982

rafaellaguimaraes.eng@gmail.com
Referencing this:

SILVA, L. M.; SANTOS, R. O. G.. Estudo comparativo: método empírico do DNIT e mecanístico-empírico (Medina) no dimensionamento de pavimentos flexíveis. Engineering Sciences, v.9, n.1, p.114-124, 2021. DOI: http://doi.org/10.6008/CBPC2318-3055.2021.001.0012 


\section{INTRODUÇÃO}

O transporte rodoviário desempenha um papel importante na sociedade e na economia brasileira, visto que tem sido a principal alternativa para a movimentação de cargas e pessoas em todo o país. Porém, para que esse transporte seja realizado de forma eficiente, faz-se necessário que o pavimento das rodovias esteja em boas condições, oferecendo economia, segurança e conforto aos usuários (CNT, 2019).

Os pavimentos podem ser divididos em três categorias sendo flexíveis, rígidos e semirrígidos. Atualmente, a maioria da malha rodoviária brasileira é composta por pavimentos flexíveis (MEZZOMO, 2014). Para atendimento dos parâmetros de projeto a via tende a ser constituída de diversas camadas onde cada uma dessas contém distintas finalidades e distinguem-se por meio das composições existentes nos materiais utilizados. Esses podem ser naturais (pedregulho, seixos, areia e brita) e artificiais (escória de aciaria, cal, cimento, argila calcinada e argila expandida).

Em situações de dimensionamento de pavimentos existem uma série de motivos para os pavimentos flexíveis apresentarem irregularidades, tais situações ocorrem devido à possível falta de planejamento e execução correta conforme as normativas abrangentes. Atualmente são notórias as imprevisibilidades quando se trata em solucionar os problemas nas pavimentações das vias brasileiras, pois costuma-se implantar sistemas corretivos e executivos a curto prazo, levando ao comprometimento das camadas constituintes e diminuindo o seu período de vida útil.

O atual método empregado no Brasil de dimensionamento de pavimentos flexíveis é executado pelo método empírico abordado pelo antigo Departamento Nacional de Estradas de Rodagem - DNER e atual Departamento Nacional de Infraestrutura de Transportes - DNIT. Foi estabelecido por meio de estudos elaborados na década de 60. Por ser antigo apresenta exceções para localidades específicas com execução em parâmetros climáticos semelhantes ao lugar que foi concebido (BERNUCCl et al., 2008).

Em contrapartida, a análise e o dimensionamento por meio do método mecanístico-empírico (M-E) trazem algumas vantagens relacionadas às metodologias empíricas de dimensionamento de pavimentos flexíveis. O método mecanístico-empírico permite um dimensionamento por meio de modelos de previsão da vida útil dos materiais empregados, ao analisar o conjunto dos materiais constituintes e considerar um conjunto de variáveis, como as características elásticas de cada material (FONSECA, 2013).

Os métodos mecanístico-empíricos se valem de conceitos da mecânica para desenvolver modelos teóricos sobre o comportamento estrutural do pavimento. O comportamento dos materiais é parametrizado por meio de conhecimento empírico, laboratorial ou de pista (BALBO, 2007).

O objetivo da presente pesquisa foi verificar a viabilidade da adoção dos métodos para dimensionamento de pavimentos flexíveis, por meio de distintos materiais empregados em bases e sub-base de pavimentos flexíveis. Entre os métodos de dimensionamento de pavimentos, o empírico atualmente empregado pelo Departamento Nacional de Infraestrutura e Transportes (DNIT) e o método mecanísticoempírico de Dimensionamento Nacional de Pavimentos (MEDINA), a fim de elucidar o uso do método mecanístico-empírico. 


\section{REVISÃO TEÓRICA}

Segundo Bernucci et al. (2008), um pavimento é uma estrutura composta por múltiplas camadas de espessuras finitas, construída sobre uma superfície final de terraplenagem. Está estrutura destina-se técnica e economicamente a resistir aos esforços provenientes do tráfego de veículos e da ação climática, ao mesmo tempo em que deve proporcionar aos usuários boas condições de rolamento, conforto, economia e segurança.

Com a alta dependência do setor rodoviário apresentada pelo país, ressalta-se a importância de um bom dimensionamento do pavimento, de forma que a estrutura seja capaz de suportar a repetição da passagem de veículos sem comprometer o mesmo com o surgimento de patologias como trincamento excessivo no revestimento asfáltico devido à fadiga, além de garantir que as outras camadas da sua estrutura sejam somadas aos materiais que as compõe diminuam os efeitos causados na resistência do pavimento pelo trânsito constante (FRANÇA, 2018).

\section{Classificação e estrutura do pavimento}

Quanto à classificação dos pavimentos, de acordo com o Manual de Pavimentação do Departamento Nacional de Infraestrutura de Transportes (DNIT, 2006), os pavimentos são classificados como: flexíveis, semirrígidos e rígidos, em conformidade com os materiais utilizados e as maneiras de divisão das cargas aplicadas, conforme descrições:

Flexíveis: destaca aqueles em que todas as camadas sofrem deformação elástica significativa sob o carregamento aplicado e, portanto, a carga se distribui em parcelas aproximadamente equivalentes entre as camadas, como se pode observar na Figura 1.

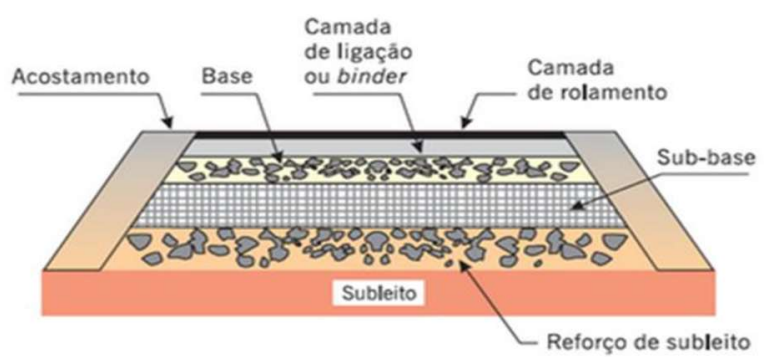

Figura 1: Estrutura De Um Pavimento Flexível. Fonte: Bernucci et al. (2008).

Semirrígidos: Como ilustrado na Figura 2 caracteriza-se por uma base cimentada por algum aglutinante com propriedades cimentícias.

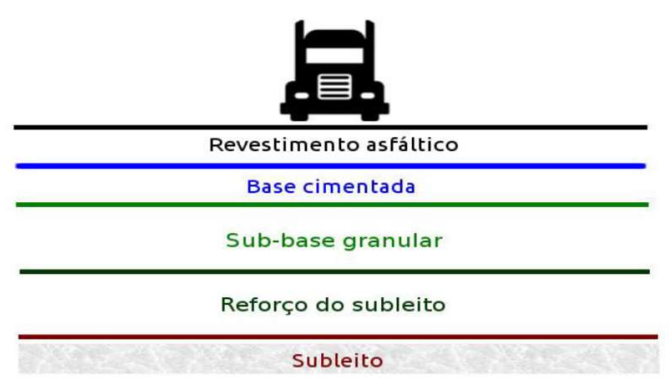

Figura 2: Estrutura do Pavimento Semirrígido. Fonte: Bernucci et al. (2008). 
Rígidos: são aqueles em que o revestimento tem uma elevada rigidez em relação às camadas inferiores e, portanto, absorve praticamente todas as tensões provenientes do carregamento aplicado, conforme a Figura 3.

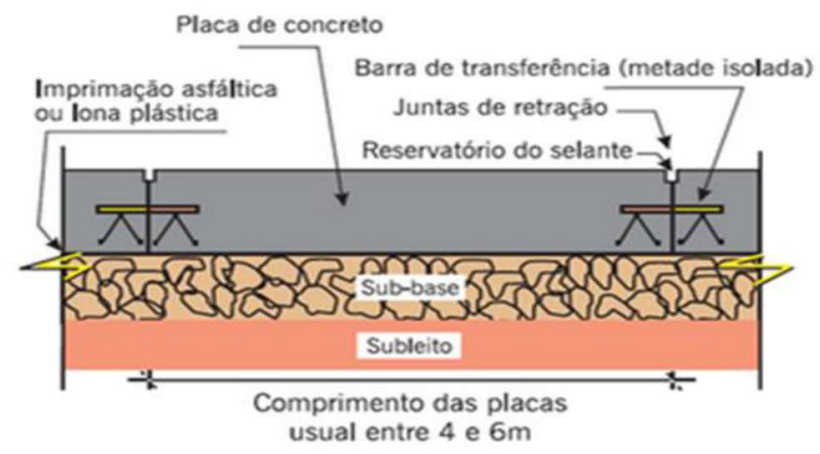

Figura 3: Estrutura de Pavimento Rígido. Fonte: Bernucci et al. (2008).

\section{Dimensionamento de pavimentos}

Conforme Balbo (2007), dimensionar um pavimento trata da determinação da espessura das camadas a serem aplicadas e a composição dos materiais que serão aplicados, pois desta maneira é possível construir uma estrutura que tenha capacidade de suportar o carregamento imposto por determinado volume de tráfego em situações climáticas do meio em que está inserido.

O dimensionamento pode partir da consideração de que as cargas aplicadas são estáticas; no entanto, ele é submetido a cargas repetidas, sofrendo, devido a essa repetição, deformações permanentes e elásticas, que serão tantas maiores, quanto maior for o número de solicitações. Embora a superfície de contato da carga de roda com o pavimento seja aproximadamente elíptica, essa superfície pode ser considerada circular (ROCHA, 2010).

Os principais mecanismos de degradação dos pavimentos são o trincamento por fadiga, oriundo das deformações elásticas, e o afundamento de trilha de roda, proveniente das deformações plásticas (MEDINA et al., 2015).

O conhecimento das técnicas construtivas é importante na definição da variabilidade dos fatores do projeto, como, por exemplo, grau de compactação, teor de umidade. Outros dados de entrada são o volume e composição do tráfego que utilizará a via e a caracterização dos materiais disponíveis na região e do subleito (MEDINA et al., 2015).

\section{Método Empírico}

O método adotado pelo DNIT foi proposto pelo engenheiro Murilo Lopes de Souza na década de 60, com base no critério do CBR, o eixo-padrão de $80 \mathrm{KN}$ é considerando o coeficiente de equivalência obtido nas pistas da AASHTO (MOTTA, 1991; MEDINA et al., 2015; BALBO, 2007).

O dimensionamento de um pavimento consiste basicamente na determinação das espessuras das camadas da estrutura desse pavimento, buscando atender o número N. Para o dimensionamento pelo método do DNIT é essencial a realização do ensaio do Índice de Suporte Califórnia (ISC) ou Califórnia BearingRatio (CBR). 
O método do DNIT utiliza ábacos que relacionam valores de tráfego com dados dos materiais do subleito para cada tipo de pavimento (TURNBULL et al., 1962).

O Índice de Suporte Califórnia é o fator de maior importância para o cálculo de qualquer pavimento. As diretrizes para realização do ensaio que determina o CBR são estabelecidas pela ME - 172 (DNIT, 2016).

Souza Júnior (2018) determinou também faixas granulométricas em que os materiais granulares devem estar para serem utilizados como camadas do pavimento, que podem observadas no Manual de Pavimentação (DNIT, 2006). Além disto, algumas considerações a respeito destas faixas devem ser consideradas como a fração passante na peneira $n^{\circ} 200$ sendo inferior a 2/3 da fração passante na peneira $\mathrm{n}^{\circ} 40$ e também a fração graúda deve apresentar um desgaste Los Angeles igual ou inferior a $50 \%$. Para materiais com experiência de uso podem-se adotar valores de desgaste maior.

Este método se baseia praticamente nas características do solo de fundação, dos materiais que o constitui, no tráfego e prevê apenas o uso do asfalto puro na pavimentação das rodovias brasileiras, não considerando as novas tecnologias adotadas atualmente para pavimentos flexíveis, também não prevendo o surgimento de possíveis patologias, como rachaduras ou trincas com o passar dos anos devido a utilização e nível de tráfego nas rodovias (FRANÇA, 2018).

\section{Método mecanistico-empírico (Software Medina)}

Franco (2007) em sua tese de doutorado, desenvolveu um método mecanístico-empírico de dimensionamento de pavimentos asfálticos, na COPPE/UFRJ, utilizando análise elástico-linear e a elástico não linear, sendo os danos estimados por modelos de previsão de fadiga, deformação permanente e deformação máxima admissível no topo do subleito.

O Medina é um software que realiza a verificação e o dimensionamento mecanístico-empírico de estruturas de pavimentos, por meio da rotina AEMC "Análise Elástica de Múltiplas Camadas". Esta rotina calcula tensões e deformações em estruturas de pavimentos sob carregamento de rodas do tipo eixo padrão rodoviário e aplica modelos de fadiga e deformação permanente para ajustar as espessuras das camadas (FRANCO, 2018).

A análise de uma estrutura de pavimento ou mesmo o seu dimensionamento requer um conjunto amplo de informações para que os resultados obtidos sejam confiáveis. As informações passam pelo conhecimento do subleito, como seu módulo e curva de deformação permanente. Outra informação que é chave para o correto funcionamento do software Medina é a definição do Número Equivalente de Eixos, o número N. Os modelos utilizados no programa Medina mostram-se sensíveis a pequenas variações do Número N, e, portanto, uma estimativa apurada é de grande importância para o sucesso do projeto (FRANCO, 2018). Segundo Nepomuceno (2019), em um projeto mecanístico-empírico são utilizados indicadores de desempenho correlacionados com os dois principais defeitos estruturais observados nos pavimentos asfálticos brasileiros: trancamentos de fadiga e afundamentos de trilhas de roda. A Figura 4 faz o resumo dos critérios e a confiabilidade de cada tipo de via. 


\begin{tabular}{|c|c|c|c|}
\hline Tipo de Via & Confiabilidade & $\begin{array}{c}\text { Área } \\
\text { Trincada }\end{array}$ & $\begin{array}{c}\text { Deformação } \\
\text { Permanente }\end{array}$ \\
\hline Sistema Arterial Principal & $95 \%$ & $30 \%$ & $10 \mathrm{~mm}$ \\
\hline Sistema Arterial Primário & $85 \%$ & $30 \%$ & $13 \mathrm{~mm}$ \\
\hline Sistema Arterial Secundário & $75 \%$ & $30 \%$ & $20 \mathrm{~mm}$ \\
\hline Sistema Coletor Primário & $85 \%$ & $30 \%$ & $13 \mathrm{~mm}$ \\
\hline Sistema Coletor Secundário & $75 \%$ & $30 \%$ & $20 \mathrm{~mm}$ \\
\hline Sistema Local & $65 \%$ & $30 \%$ & $20 \mathrm{~mm}$ \\
\hline
\end{tabular}

Figura 4: Critério e confiabilidade de cada tipo de via. Fonte: Franco (2018).

Observa-se, portanto, que o método mecanístico-empírico em que o Medina é baseado, realiza o dimensionamento buscando minimizar a fadiga do revestimento (trincamento) e a deformação das camadas do pavimento (afundamento).

\section{METODOLOGIA}

Para a realização da pesquisa foram efetuadas análises que envolveram preliminarmente a coleta de informações por meio de pesquisas bibliográficas em trabalhos relacionados ao assunto, como em artigos, revistas, congressos da área de pavimentação, normativas, averiguações na internet e jornais, a fim de conferir uma investigação técnica e crítica dos métodos para dimensionamento de pavimentos flexíveis.

Para prosseguimento da próxima etapa envolveu a simulação de um pavimento utilizando os dois métodos. Para tal, os materiais utilizados no dimensionamento e seus parâmetros foram adotados com base nos resultados obtidos por Rocha et al. (2017). Tal pesquisa foi utilizada por ter realizado ensaios de Índice de Suporte Califórnia (ISC) e Módulo de Resiliência (MR) com diferentes materiais, possibilitando utilizar um mesmo material no dimensionamento com o atual método do DNIT, que utiliza ISC, e com o Medina, que necessita de MR.

Logo em seguida a análise dos dados obtidos pelo método do DNIT, realizou-se o preenchimento do software Medina, com dados e as informações das composições constituintes de cada material abordado. 0 software é o responsável pelo cálculo das espessuras do pavimento, sendo considerado um método mecanístico-empírico, onde considera os fatores climáticos e os tipos dos materiais da localidade.

\section{Dados de projeto}

Para o proposto dimensionamento foram considerados volumes de tráfego diferentes. Com especificações do Manual do Departamento Nacional de Infraestrutura de Transporte - (DNIT, 2006) adotouse como carga padrão 8,2T por eixo para tráfego leve e médio com período de projeto de 10 anos. 0 caso 1 considerou o número $\mathrm{N}=10^{6}$ e o caso 2 considerou $\mathrm{N}=10^{7}$. Para tal, em $\mathrm{N}=10^{6}$, considerou-se sistema coletor secundário para o tipo da via, onde deve obedecer no Medina aos padrões característicos de $75 \%$ de confiabilidade e com área trincada dentro dos $30 \%$ permitido, adotou-se VMD de 274 . Para $N=10^{7}$, considerou-se sistema coletor primário, com VDM de 1370, devendo obedecer no Medina aos padrões característicos de confiabilidade de $85 \%$ e área trincada com $30 \%$.

Para os materiais de base e sub-base, como já comentado, os dados adotados foram conforme 
resultados obtidos por Rocha et al. (2017), que abordaram distintos materiais e dosagens envolvendo cascalho - C, areia - A, cal hidratada - CA e cimento - $\mathrm{Cl}$. Os dados adotados podem ser observados na Tabela 1.

Tabela 1: Dados dos materiais constituintes das camadas de base e sub-base.

\begin{tabular}{lllcll}
\hline Camada & Material & Coeficiente Estrutural (K) & $\begin{array}{l}\text { CBR } \\
\text { (\%) }\end{array}$ & $\begin{array}{l}\text { Módulo de Resiliência } \\
\text { (MPa) }\end{array}$ & Coeficiente de Poisson \\
\hline Base & $\mathrm{C}+20 \% \mathrm{~A}+2 \% \mathrm{Cl}$ & 1,7 & 144 & $\mathrm{MR}=308$ & 0,35 \\
\hline Sub-base & $\mathrm{C}+6 \% \mathrm{CA}$ & 1 & 48 & $\mathrm{MR}=257$ & 0,35 \\
\hline
\end{tabular}

Fonte: Adaptada Rocha et al. (2017).

Para o revestimento, o método empírico contempla Tratamentos Superficiais, Concreto Betuminoso, pré-misturado a quente e pré-misturado a frio. O software Medina tem outras variações como, por exemplo, concreto asfáltico modificado, concreto asfáltico borracha e diferentes classes de concreto asfáltico usinado a quente.

\section{RESULTADOS E DISCUSSÃO}

O dimensionamento do pavimento utilizando os dois métodos teve como resultado o tipo de revestimento e as espessuras das camadas, sendo que para base e sub-base os materiais foram adotados conforme bibliografia de Rocha et al. (2017). Os resultados obtidos podem ser observados na Tabela 2.

Tabela 2: Dados obtidos pelo método empírico do DNIT e do mecanístico-empírico utilizando o software Medina

\begin{tabular}{|c|c|c|c|c|c|c|c|}
\hline \multirow{2}{*}{$\begin{array}{l}\text { Tráfego } \\
\text { Método }\end{array}$} & \multicolumn{4}{|l|}{$\mathrm{N}=1 \times 10^{6}$} & \multicolumn{3}{|l|}{$\mathrm{N}=1 \times 10^{7}$} \\
\hline & $\begin{array}{l}\text { Empírico } \\
\text { (DNIT) }\end{array}$ & ME (Medina) & & & $\begin{array}{l}\text { Empírico } \\
\text { (DNIT) }\end{array}$ & ME (Medina) & \\
\hline Caso & Caso 1 & Caso 1.1 & Caso 1.2 & Caso 1.3 & Caso 2 & Caso 2.1 & Caso 2.2 \\
\hline \multirow{6}{*}{ Revestimento } & Tratamento & Tratamento & Concreto & Concreto & Concreto & Concreto asfáltico & Concreto \\
\hline & Superficial & Superficial & Asfáltico & Asfáltico & Betuminoso & modificado (CAM & asfáltico \\
\hline & & (Duplo - MR & Borracha (CAM & (Classe 1 - MR & & RJ CAPFLEX MR & (classe $4-\mathrm{MR}$ \\
\hline & & $1000 \mathrm{MPa})$ & RJ CAPFLEX - MR & $5764 \mathrm{MPa})$ & & $3184 \mathrm{MPa})$ & $10492 \mathrm{MPa})$ \\
\hline & & & $3184 \mathrm{MPa})$ & & & & \\
\hline & - & $2 \mathrm{~cm}$ & $10 \mathrm{~cm}$ & $5 \mathrm{~cm}$ & $7,5 \mathrm{~cm}$ & $15 \mathrm{~cm}$ & $9,4 \mathrm{~cm}$ \\
\hline Base & $15 \mathrm{~cm}$ & $15 \mathrm{~cm}$ & $15 \mathrm{~cm}$ & $15 \mathrm{~cm}$ & $15 \mathrm{~cm}$ & $15 \mathrm{~cm}$ & $15 \mathrm{~cm}$ \\
\hline Sub-base & $15 \mathrm{~cm}$ & $15 \mathrm{~cm}$ & $15 \mathrm{~cm}$ & $15 \mathrm{~cm}$ & $15 \mathrm{~cm}$ & $15 \mathrm{~cm}$ & $15 \mathrm{~cm}$ \\
\hline
\end{tabular}

O dimensionamento realizado para os distintos casos utilizou os mesmos parâmetros para base e sub-base e com os dados obtidos, verifica-se que todas as tentativas apresentaram resultados semelhantes. Sucedeu devido serem considerados os mesmos parâmetros dos materiais para as camadas de base e subbase, houve alteração somente no tipo de revestimento, permitindo assim a variação em sua espessura. Vale ressaltar que o software Medina permite diversas tentativas em tempo hábil, podendo variar desde os materiais constituintes das camadas, adicionar camadas ou suprimi-las, podendo verificar o dimensionamento novamente, gerando novos cenários e simulações. Na presente pesquisa, optou-se por manter camada de base e sub-base em todos os casos.

Para a primeira simulação, atribuiu-se o valor de VDM de 274, apresentando o número $\mathrm{N}$ minimamente maior do que $1 \times 10^{6}\left(1,0001 \times 10^{6}\right)$. No software Medina, tal valor apresenta incompatibilidade com o programa para utilização de Tratamento Superficial como revestimento, aparecendo uma mensagem 
de alerta informando que não se pode utilizar tratamento superficial duplo para tráfegos com o volume adotado. Foi efetuada uma nova tentativa com o VMD 273 buscando atingir o número N próximo de $1 \times 10^{6}$ para período de projeto de 10 anos, obtendo assim o afundamento da trilha de roda em 4,2mm e na análise realizada não considerou o dano relativo à fadiga, como mostra a Figura 5.

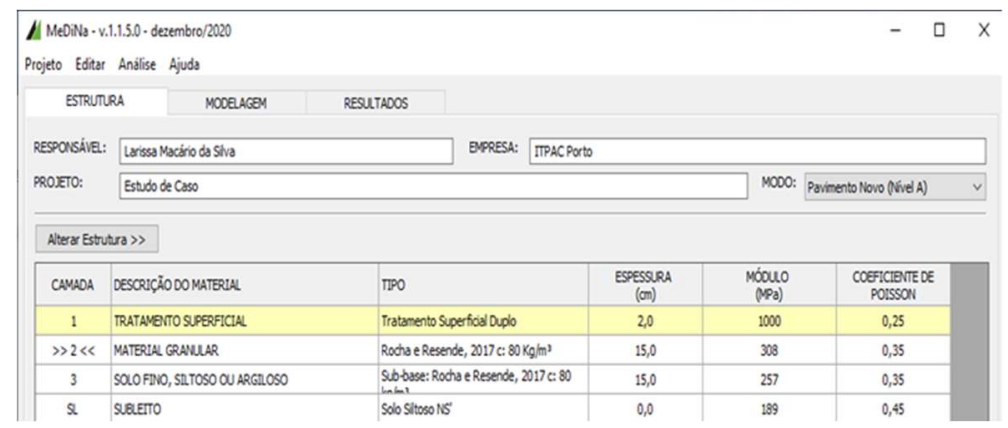

Figura 5: Dimensionamento do caso 1.1.

O dimensionamento no Medina considerando $N=10^{6}$ foi refeito, considerando outros revestimentos, chamando de caso 1.2 Adotando concreto asfáltico borracha, de acordo com a Figura 6, obteve $3,5 \%$ de área trincada do pavimento, afundamento da trilha de roda em 3,2mm.

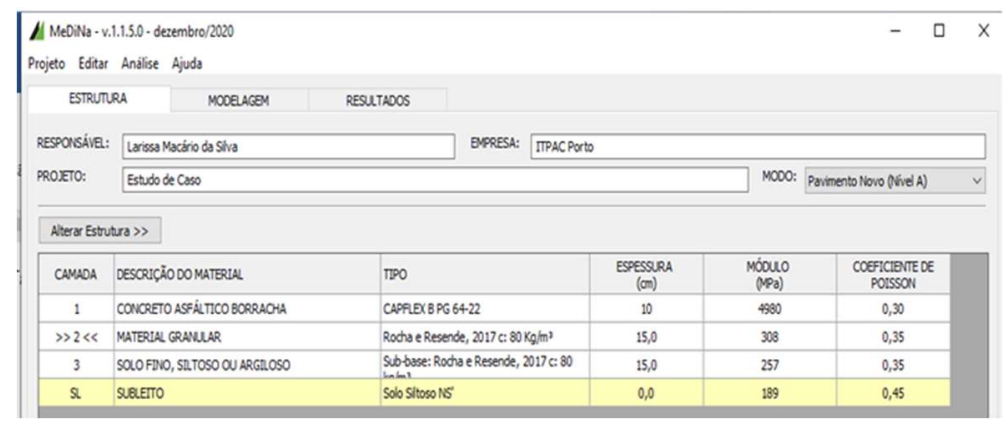

Figura 6: Dimensionamento do caso 1.2.

Por meio do critério de confiabilidade ilustrado na Figura 7 a análise efetuada para o tráfego de $\mathrm{N} \times 10^{6}$ de acordo como caso 1.3 obteve-se 3,5\% de área trincada do pavimento, afundamento da trilha de roda em $3,2 \mathrm{~mm}$.

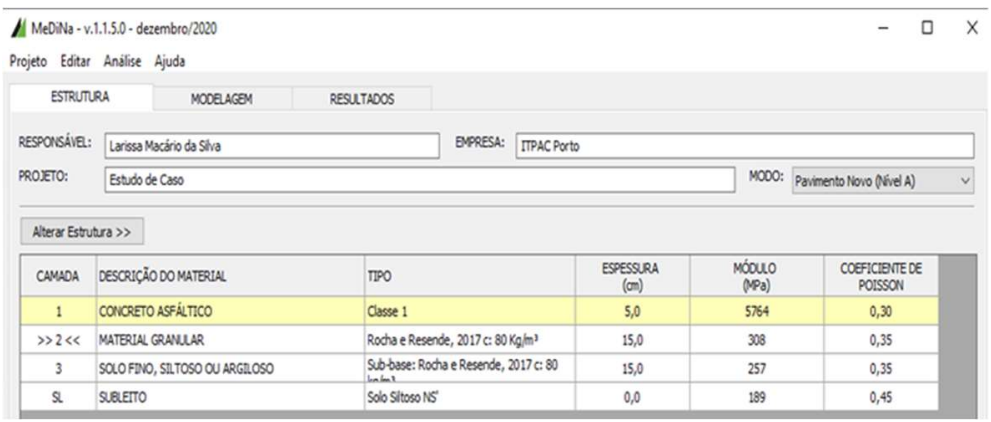

Figura 7:Dimensionamento do caso 1.3.

O critério de confiabilidade para o caso 2.1, apresentou a análise efetuada pelo programa, área trincada com $29,2 \%$, afundamento da trilha de roda em $3,7 \mathrm{~mm}$, como mostra a Figura 8. 


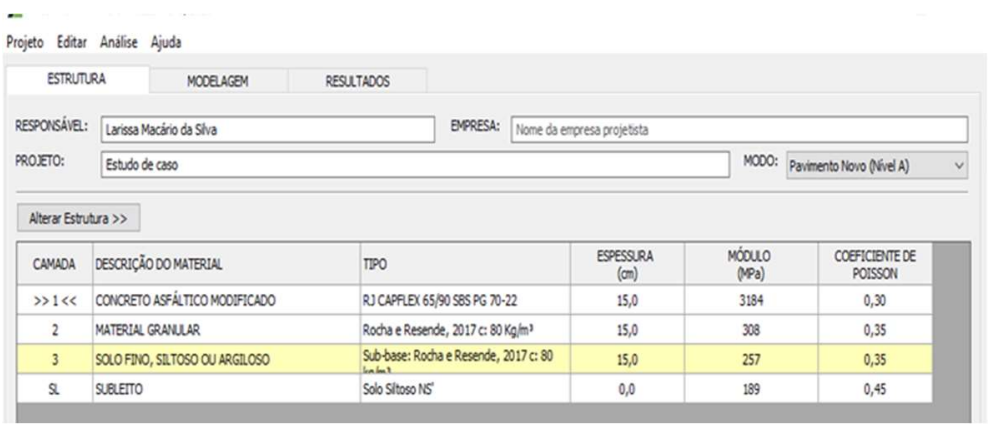

Figura 8: Dimensionamento do caso 2.1.

De acordo com a Figura 9, o critério de confiabilidade para o caso 2.2, apresentou a análise efetuada pelo programa, área trincada com $28,8 \%$ de área trincada do pavimento, o afundamento da trilha de roda em $3,7 \mathrm{~mm}$.

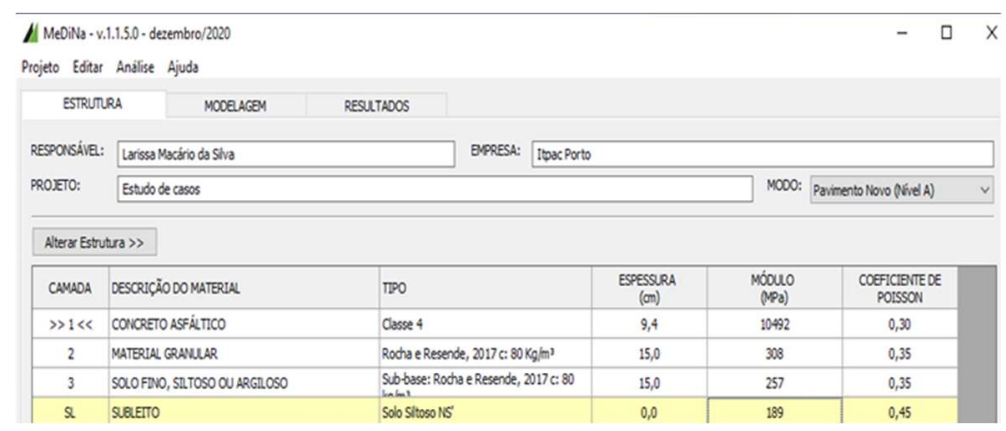

Figura 9: Dimensionamento do caso 2.2.

Observa-se que para o tráfego considerado de $10^{6}$, a diferença da estrutura do pavimento dimensionado nos dois métodos foi no revestimento, uma vez que no método empírico o Tratamento Superficial foi suficiente (Caso 1), enquanto no mecanístico-empírico o afundamento na trilha de roda foi de 4,2 $\mathrm{mm}$ para TSD e utilizando outros revestimentos passou a ser de 3,2 $\mathrm{mm}$.

Neste caso, vale ressaltar a diferença dos valores de módulo de tais revestimentos. Para os materiais disponíveis no software, enquanto o tratamento superficial tem valor de módulo resiliente de $1000 \mathrm{MPa}$ para TSD e 1500 MPa para TST, os módulos de concreto asfáltico partem de 5764 MPa.

Considerando o tráfego com número $\mathrm{N}$ de $10^{7}$, mais uma vez a diferença observada foi no revestimento, sendo nesses casos espessuras significativas. Utilizando o método empírico, a espessura indicada é de no mínimo $7,5 \mathrm{~cm}$, sendo que deveria ser de $10 \mathrm{~cm}$ caso o número $\mathrm{N}$ fosse maior do que $10^{7} \mathrm{e}$ menor ou igual a $5 \times 10^{7}$. Vale lembrar que o método empírico não leva em consideração a variedade de revestimentos que existem atualmente, que poderiam diminuir a espessura e/ou baratear o revestimento. Já se utilizando o software Medina, os revestimentos, no caso 1.1: $2 \mathrm{~cm}$, caso 1.3: $10 \mathrm{~cm}$, caso 1.3: $5 \mathrm{~cm}$ no segundo caso 2.1: $15 \mathrm{~cm}$ e no caso 2.2: 9,4 cm, efetuando alteração no material do revestimento foi possível verificar a modificação nas espessuras da camada, isso se deve aos valores dos módulos resilientes adotados.

Nos dimensionamentos elaborados pelo método DNIT (2006), pode-se averiguar uma diferença nas camadas dos revestimentos para ambos os casos, nas demais atingiu-se as mesmas espessuras. 


\section{CONCLUSÕES}

Os resultados das análises obtidas no levantamento bibliográfico foram satisfatórios, visto que objetivo geral dessa pesquisa foi alcançado, designando medidas sobre as comparações entre os específicos métodos abordados nesta pesquisa.

O método mecanístico-empírico defronta-se com certa resistência ao seu uso no meio profissional da área da engenharia, ao se observar que o ensaio de módulo de resiliência dos materiais é considerado de maior dificuldade por depender de alguns fatores como, por exemplo, a necessidade de equipamentos especializados. Entretanto, o método do Medina apresenta certa confiabilidade se comparado ao método empírico do DNIT, uma vez que permite a utilização de maiores fontes de dados de cada material, possibilitando alternância nas composições através dos ensaios conforme características envolvendo o clima da região em estudo, sendo este limitado no método do DNIT. Outra condição favorável é a consideração da exploração da qualidade das misturas asfálticas para prever a ação da fadiga.

Entretanto, os resultados obtidos manifestaram que houve gradativa alteração nas espessuras comparada pelos métodos propostos, se tratando da divergência somente na camada de revestimento quando se modificou o material, sendo este responsável por influenciar na espessura final da camada, fator diretamente ligado ao módulo resiliente, no dimensionamento realizado nos dois casos as espessuras das camadas foram menores quando houve o aumento do módulo resiliente do material constituinte do revestimento. Sendo que as demais camadas atingiram os mesmos valores tanto para a camada de base quando sub-base em ambos os métodos.

\section{REFERÊNCIAS}

BALBO, J. T.. Pavimentação asfáltica: materiais, projeto e restauração. São Paulo: Oficina de Textos, 2007.

BERNUCCI, L. B.; MOTTA, L. M. G.; CERATTI, J. A. P.; SOARES, J. B.. Materiais e estruturas de pavimentos asfálticos. In: Pavimentação asfáltica: formação básica para engenheiros. Rio de Janeiro: PETROBRAS-ABEDA, 2008. p.79-110.

CNT. Confederação Nacional do Transporte. Condições das Rodovias. Brasília: CNT, 2019.

DNIT. Departamento Nacional de Infraestrutura de Transportes. Manual de Pavimentação. 3 ed. Rio de Janeiro: DNIT, 2006.

FONSECA, L. F. S.. Análise das soluções de pavimentação do programa CREMA 2a Etapa do Departamento Nacional de Infraestrutura de Transportes. Dissertação (Mestrado em Engenharia Civil) - Universidade Federal do Rio de Janeiro, Rio de Janeiro, 2013.

FRANÇA, A. C. P.. Estudo comparativo entre o método de dimensionamento de pavimentos e o novo método Medina. Monografia (Bacharelado em Engenharia Civil) Universidade de Ceuma, São Luiz, 2018.

FRANCO, A. C. P.. Análise elástica de múltiplas camadas. Manual de Utilização. Versão 2.4. Rio de Janeiro, 2018.
FRANCO, A. C. P.. Método de dimensionamento Mecanístico-empírico de Pavimentos asfálticos - SisPav. Tese (Doutorado) - Universidade Federal do Rio de Janeiro, Rio de Janeiro, 2007.

MEDINA, J.; MOTTA, L. M. G.. Mecânica dos Pavimentos. 3 ed. Rio de Janeiro: Interciência, 2015.

MEZZOMO, H.. Análise comparativa entre um pavimento de concreto simples e um flexível para a duplicação da Rodovia $\mathrm{Br}-386 /$ Rs. Monografia (Bacharelado em Engenharia Civil) - Centro Universitário Univates, Lajeado, 2014.

MOTTA, L. M. G.. Método de dimensionamento de pavimentos flexíveis; critério de confiabilidade e ensaios de cargas repetidas. Tese (Doutorado) - Universidade Federal do Rio de Janeiro, Rio de Janeiro, 1991.

NEPOMUCENO, D. V.. Avaliação em campo de estrutura de pavimento incorporado solo estabilizado com escória KR sujeita à carregamento com simulador HVS e comparação com previsões de desempenho segundo o novo método brasileiro de dimensionamento Medina. Dissertação (Mestrado em Engenharia Civil) - Universidade Federal do Espírito Santo, Vitória, 2019. 
ROCHA, A. J.. Dimensionamento de pavimentos flexíveis: comparação dos métodos empírica e Mecanístico.

Monografia (Bacharelado em Engenharia Civil) -

Universidade Estadual de Feira de Santana, Feira de Santana, 2010.

ROCHA, M. T.; RESENDE, L. R.. Estudo laboratorial de um solo tropical granular estabilizado quimicamente para fins de pavimentação. Dissertação (Mestrado em Engenharia Civil) - Universidade Federal de Goiás, Goiânia, 2017.
SOUZA JÚNIOR, J. G. Aplicação do novo método de dimensionamento de pavimentos asfálticos a trechos de uma rodovia federal. Dissertação (Mestrado em Engenharia Civil) - Universidade Federal do Rio de Janeiro, Rio de Janeiro, 2018.

TURNBULL, W. J.; FOSTER, C. R.; AHLVIN, R. G.. Design of flexible pavements considering mixed loads and traffic. In INTERNATIONAL CONFERENCE ON STRUCTURAL DESIGN OF ASPHALT PAVEMENTS, 1. Annals. Lansing, 1962.

A CBPC - Companhia Brasileira de Produção Científica (CNPJ: 11.221.422/0001-03) detém os direitos materiais desta publicação. Os direitos referem-se à publicação do trabalho em qualquer parte do mundo, incluindo os direitos às renovações, expansões e disseminações da contribuição, bem como outros direitos subsidiários. Todos os trabalhos publicados eletronicamente poderão posteriormente ser publicados em coletâneas impressas sob coordenação da Sustenere Publishing, da Companhia Brasileira de Produção Científica e seus parceiros autorizados. Os (as) autores (as) preservam os direitos autorais, mas não têm permissão para a publicação da contribuição em outro meio, impresso ou digital, em português ou em tradução. 